University of Wollongong

Research Online

Faculty of Engineering and Information

Faculty of Engineering and Information

Sciences - Papers: Part A

Sciences

$1-1-2020$

\title{
Effect of quantum reflection over the barrier on thermionic refrigeration
}

Zeyu Zhang

Zelong Peng

Zhongshui Ma

C Zhang

University of Wollongong, czhang@uow.edu.au

Follow this and additional works at: https://ro.uow.edu.au/eispapers

Part of the Engineering Commons, and the Science and Technology Studies Commons

Research Online is the open access institutional repository for the University of Wollongong. For further information contact the UOW Library: research-pubs@uow.edu.au 


\title{
Effect of quantum reflection over the barrier on thermionic refrigeration
}

\author{
Abstract \\ (c) 2020 Author(s). We study the effect of quantum reflection over the barrier (ROB) in a thermionic cooling \\ device. We find that the performance of refrigerators can be enhanced by the ROB effect if the bias \\ voltage and the lattice thermal resistance of the semiconductor in the barrier region are both sufficiently \\ high. Furthermore, the figure of merit Z T can be higher due to the ROB effect if the workfunction of the \\ cathode is low and the lattice thermal resistance is high. The overall optimum $Z \mathrm{~T}$ calculated with and \\ without the ROB effect are 6.5 and 7.1, respectively. The origin of the ROB correction to $\mathrm{ZT}$ is that the \\ quantum reflection becomes asymmetric for the carrier transport in opposite directions.

\section{Disciplines} \\ Engineering | Science and Technology Studies

\section{Publication Details} \\ Zhang, Z., Peng, Z., Ma, Z. \& Zhang, C. (2020). Effect of quantum reflection over the barrier on thermionic \\ refrigeration. Journal of Applied Physics, 128 (4),
}




\section{Effect of quantum reflection over the barrier on thermionic refrigeration}

Cite as: J. Appl. Phys. 128, 044301 (2020); https://doi.org/10.1063/5.0011059

Submitted: 17 April 2020 . Accepted: 14 July 2020 . Published Online: 29 July 2020

Zeyu Zhang, Zelong Peng (D), Zhongshui Ma, and Chao Zhang (D)
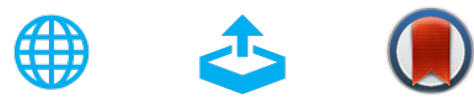

View Online

Export Citation

\section{ARTICLES YOU MAY BE INTERESTED IN}

Limiting output voltage of isentropic energy-emissive harvesters

Journal of Applied Physics 128, 044501 (2020); https://doi.org/10.1063/5.0006535

Theoretical efficiency limit of graphene-semiconductor solar cells

Applied Physics Letters 117, 053902 (2020); https://doi.org/10.1063/5.0020080

Feature engineering of material structure for Al-based materials knowledge systems

Journal of Applied Physics 128, 041103 (2020); https://doi.org/10.1063/5.0011258

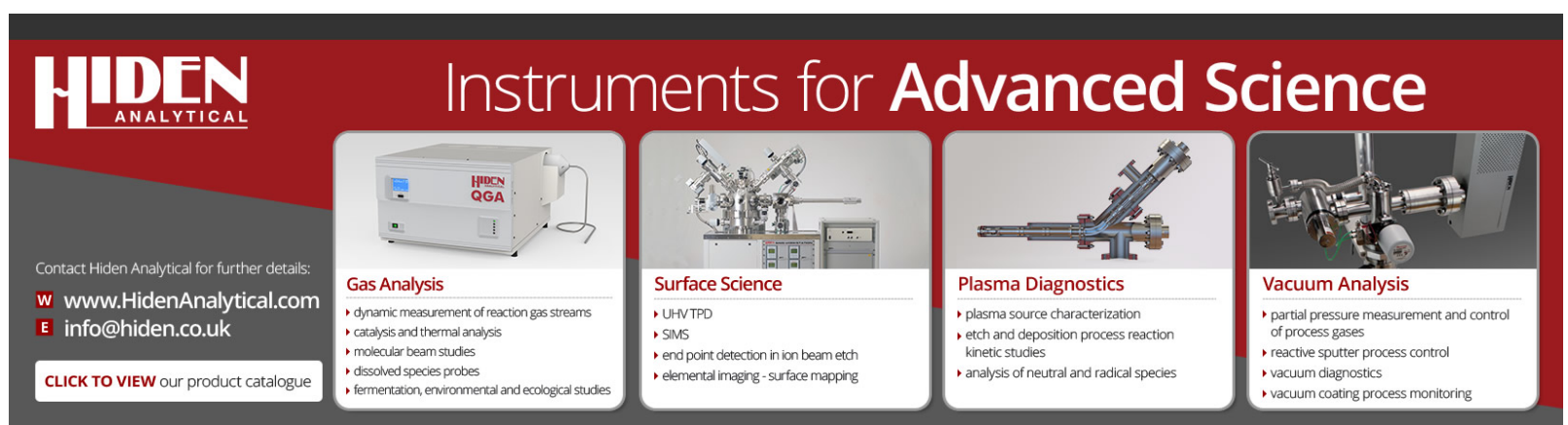




\title{
Effect of quantum reflection over the barrier on thermionic refrigeration
}

\author{
Cite as: J. Appl. Phys. 128, 044301 (2020); doi: 10.1063/5.001 1059 \\ Submitted: 17 April 2020 - Accepted: 14 July 2020 . \\ Published Online: 29 July 2020
}

Zeyu Zhang, ${ }^{1, a)}$ Zelong Peng, ${ }^{1}$ (ID Zhongshui Ma, ${ }^{1,2}$ and Chao Zhang ${ }^{3, a)}$ (iD)

\author{
AFFILIATIONS \\ ${ }^{1}$ School of Physics, Peking University, Beijing 100871, China \\ ${ }^{2}$ Collaborative Innovation Center of Quantum Matter, Beijing 100871, China \\ ${ }^{3}$ School of Physics, University of Wollongong, New South Wales 2522, Australia
}

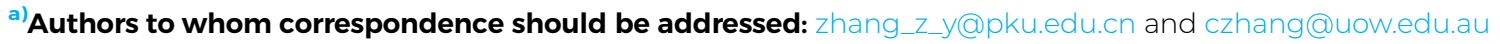

\begin{abstract}
We study the effect of quantum reflection over the barrier (ROB) in a thermionic cooling device. We find that the performance of refrigerators can be enhanced by the ROB effect if the bias voltage and the lattice thermal resistance of the semiconductor in the barrier region are both sufficiently high. Furthermore, the figure of merit $Z T$ can be higher due to the ROB effect if the workfunction of the cathode is low and the lattice thermal resistance is high. The overall optimum $Z T$ calculated with and without the ROB effect are 6.5 and 7.1, respectively. The origin of the $\mathrm{ROB}$ correction to $Z T$ is that the quantum reflection becomes asymmetric for the carrier transport in opposite directions.
\end{abstract}

Published under license by AIP Publishing. https://doi.org/10.1063/5.0011059

\section{INTRODUCTION}

Thermionic refrigerators (power generators) have attracted considerable attention recently due to their potential for reliable, environmentally friendly, scalable and high-efficiency operation. ${ }^{1-13}$ Thermionic emission is a process where electrons are thermally emitted from the surface of materials. The current density $J$ of thermionic emission is determined by the well-known RichardsonDushman (RD) law, ${ }^{14}$

$$
J_{e}=A T^{2} \exp \left(-\frac{\Phi_{0}}{k_{B} T}\right)
$$

where $T$ is the absolute temperature in Kelvin, $\Phi_{0}$ is the workfunction of the material in electronvolts, $k_{B}$ is the Boltzmann constant, $A=4 \pi e m_{e} k_{B}^{2} / h^{3}=120 \mathrm{~A} \mathrm{~cm}^{-2} \mathrm{~K}^{-2}$ is the Richardson constant, $e$ is the electron charge, $m_{e}$ is the electron mass, and $h$ is the Planck constant. For convenience, we set electron charge $e>0$, here and hereafter, and ignore the minus sign as if all carriers in our system are holes rather than electrons.

A typical vacuum-state thermionic refrigerator ${ }^{14}$ (VSTR) consists of two electrodes separated by a small vacuum gap. In a solid-state thermionic refrigerator ${ }^{15-17}$ (SSTR), the gap between two electrodes is filled with a solid, usually a semiconductor.
The electrodes can be either metals or semiconductors. Many different semiconductors have been investigated for use in SSTR, including AlGaAs, ${ }^{18} \mathrm{SiGeC} / \mathrm{Si},{ }^{19,20} \mathrm{Hg}_{1-x} \mathrm{Cd}_{x} \mathrm{Te}^{21}$ and $\mathrm{InP} .{ }^{22}$ In a thermionic cooling process, thermally activated electrons with energy greater than the workfunction are emitted from one electrode and travel over the energy barrier to reach the opposite electrode. The net electrical and heat flux across the barrier can be controlled by an applied bias voltage and a temperature difference between two electrodes. Cooling can be achieved when the applied bias exceeds a threshold value.

The performance of a refrigerator is given by the coefficient of performance (COP), defined $\mathrm{ss}^{23}$

$$
\mathrm{COP}=\frac{Q_{1}}{W}
$$

where $Q_{1}$ is the heat extracted from the low temperature reservoir and $W$ is the work input by an external source.

The main advantage of SSTRs is that the device can have a much lower workfunction. This is because the effective barrier height in a SSTR is the difference between the cathode's workfunction and the solid's electron affinity. Some schemes using negative electron affinity to increase the COP have been proposed. ${ }^{24-26}$ Another disadvantage of VSTR is the space-charge effect which 
increases the effective workfunction and reduces the output. In a SSTR, this effect is much less significant as compared to a VSTR since we can design the barrier width (the width of a solid layer) to be much smaller than the vacuum gap in VSTR. ${ }^{14}$ On the other hand, there is a significant heat backflow in a SSTR (from the hot electrode to the cold one) due to the finite thermal conduction (lattice thermal conductivity) in the barrier layer which is a layer of semiconductor in our system. The heat backflow reduces the COP. In recent years, the thermionic emission and corresponding refrigerators (power generators) have been studied in many different ways such as by using two dimensional materials, ${ }^{2,3,6,27-33}$ photon-enhanced thermionic emission, ${ }^{7,8,34-40}$ and van der Waals heterostructures. ${ }^{27-29}$

In a thermionic process, the electrons traverse the barrier ballistically without loss of energy. In a thermoelectric process, electrons traverse the conductor diffusively. We now linearize the electrical current $J_{e c}$ and thermal current $J_{q c}$ with regard to that bias gradient $\nabla v$ and temperature gradient $\nabla T$ in a thermionic process,

$$
\begin{aligned}
& J_{e c}=\mathcal{L}_{11}^{0}(\nabla v)+\mathcal{L}_{12}^{0}(-\nabla T), \\
& J_{q c}=\mathcal{L}_{21}^{0}(\nabla v)+\mathcal{L}_{22}^{0}(-\nabla T) .
\end{aligned}
$$

By using this linearization, we can make an analogy between the thermionic and the thermoelectric process, that is, we can define corresponding "thermoelectric" quantities in a thermionic process, ${ }^{15}$

$$
\begin{gathered}
\sigma=\mathcal{L}_{11}^{0}, \\
S=\frac{\mathcal{L}_{12}^{0}}{\mathcal{L}_{11}^{0}}, \\
\kappa=\frac{\mathcal{L}_{22}^{0} \mathcal{L}_{11}^{0}-\left(\mathcal{L}_{12}^{0}\right)^{2}}{\mathcal{L}_{11}^{0}},
\end{gathered}
$$

where $\sigma$ is the electrical conductivity, $S$ is the Seebeck coefficient, and $\kappa$ is the thermal conductivity. The performance of a thermoelectric or a thermionic device can be characterized by the figure of merit $Z T,{ }^{41,42}$ given as

$$
Z T=\frac{\sigma S^{2} T}{\kappa},
$$

where $T$ is the absolute temperature. The higher the $Z T$ is, the better the device performs.

As mentioned above, the charge transport is mainly due to over barrier hopping of hot electrons in a thermionic process. In a classical description, it is usually assumed that the transport is ballistic and the transmission over the barrier is $100 \%$. However, if the electrons are regarded as waves, the reflection at boundary is finite and the transmission will be less than $100 \%$. The purpose of this paper is to analyze the effect of the reflection over the barrier (ROB) on a thermionic cooling process. This pure quantum effect is expected to be small and the fact that RD law works well suggests that $\mathrm{ROB}$ may have a negligible effect on the emission process. However, for a thermionic device with two electrodes, the effect of
$\mathrm{ROB}$ on the charge and heat transport along opposite directions is not the same. Thus, while the ROB suppresses the emission, it can either reduce or enhance the overall performance of the device. Therefore, it is important to have a quantitative understanding of the quantum effect.

In this paper, we show quantitatively how the ROB influences the COP and $Z T$ of a thermionic device. We focus on the difference between two models: with and without the ROB effect. The result of the model without the ROB effect is already known. ${ }^{15,16}$ The $\mathrm{ROB}$ effect is analyzed in this work and the results are as follows. Generally, due to the ROB effect, transport probability is less than the classical value of $100 \%$, and the ROB slightly reduces the electric current and the thermal current. However, due to the inequivalent ROB effect in the two electrodes, we find that the COP and $Z T$ can be higher due to the ROB effect in some regions of workfunction and thermal resistance. The optimum bias (workfunction) of COP (ZT) is slightly greater (smaller) due to the ROB effect. The overall optimum ZT is smaller when the ROB effect is included. These findings may be useful in designing and developing highefficiency thermionic cooling devices.

\section{THERMIONIC PROPERTIES OF A SINGLE BARRIER STRUCTURE}

\section{A. Electric current and thermal current}

We consider a single barrier structure shown in Fig. 1, where the cathode and the anode are separated by a semiconductor of thickness $d$. Here, for simplicity, we assume the same workfunction for the cathode and the anode. A bias voltage is applied to drive the current from the cold to hot electrode. We ignore the space charge

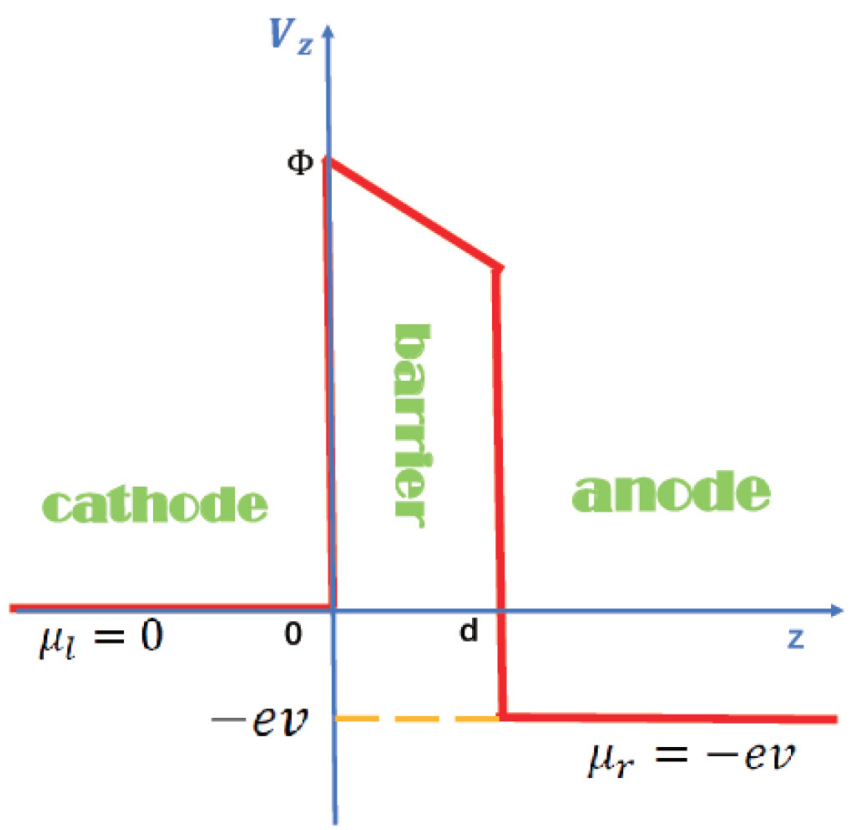

FIG. 1. Schematic of a single barrier thermionic structure an applied bias. 
effect and band bending so that the electric field between two electrodes can be viewed as uniform. This assumption is proper because when semiconductor thickness is small enough, the space charge effect can be ignored ${ }^{14}$ and the band bending can be minimized by adjusting the chemical potential of the semiconductor. ${ }^{9,43}$ We choose the position of the cathode/barrier interface as the origin of coordinates and the cathode chemical potential is the zero energy point as shown in Fig. 1. The potential can be written as

$$
V(z)= \begin{cases}0, & (z<0) \\ \Phi-\frac{e v}{d} z, & (0 \leq z \leq d) \\ -e v, & (z>d)\end{cases}
$$

where $\Phi$ is the workfunction of cathode, $v$ is the bias voltage, and $d$ is the barrier thickness. For an electron incident from the left electrode, the wave function can be written as

$$
\psi(z)= \begin{cases}e^{i k_{1} z}+R_{l} e^{-i k_{1} z}, & (z<0), \\ A \psi_{1}(\xi(z))+B \psi_{2}(\xi(z)), & (0<z<d), \\ T_{l} e^{i k_{2} z}, & (z>d),\end{cases}
$$

where $k_{1}=\sqrt{2 m E_{z}} / \hbar$ and $k_{2}=\sqrt{2 m\left(E_{z}+e v\right)} / \hbar$ are the wavevectors of the wave function in the regions of $z<0$ and $z>d$, respectively. $\psi_{1}(\xi(z))$ and $\psi_{2}(\xi(z))$ are given as

$$
\psi_{1}(\xi(z))=\xi(z)^{\frac{1}{2}} H_{\frac{1}{3}}^{(1)}\left(\frac{2}{3} \xi(z)^{\frac{3}{2}}\right)
$$

and

$$
\psi_{2}(\xi(z))=\xi(z)^{\frac{1}{2}} H_{\frac{1}{3}}^{(2)}\left(\frac{2}{3} \xi(z)^{\frac{3}{2}}\right)
$$

where $H_{1}^{(1)}$ and $H_{1}^{(2)}$ stand for the first kind and the second kind of Hankkel function, ${ }^{44}$ respectively, and $\xi(z)=$ $\left[2 m e v /\left(\hbar^{2} d\right)\right]^{1 / 3}\left[z+\left(E_{z}-\Phi\right) d /(e v)\right]$ is a dimensionless parameter.

From the boundary conditions that $\psi$ and its derivative being continuous at $z=0$ and $z=d$, we find

$$
R_{l}=\frac{1+i \frac{\eta_{l} \psi_{1}^{\prime}(\xi(0))+\psi_{2}^{\prime}(\xi(0))}{k_{1}\left[\eta_{l} \psi_{1}(\xi(0))+\psi_{2}(\xi(0))\right]}}{1-i \frac{\eta_{l} \psi_{1}^{\prime}(\xi(0))+\psi_{2}^{\prime}(\xi(0))}{k_{1}\left[\eta_{l} \psi_{1}(\xi(0))+\psi_{2}(\xi(0))\right]}},
$$

where

$$
\eta_{l}=-\frac{\psi_{2}^{\prime}(\xi(d))-i k_{2} \psi_{2}(\xi(d))}{\psi_{1}^{\prime}(\xi(d))-i k_{2} \psi_{1}(\xi(d))}
$$

In above equations, derivatives are with respect to $z$, not $\xi(z)$.

The reflection coefficient is given as $r_{l}=\left|R_{l}\right|^{2}$. The electric and the thermal currents is given as $J_{\alpha}$ and $J_{Q_{\alpha}}$, where $\alpha=l(r)$ for the current from left(right) electrode the right(left) electrode,

$$
J_{\alpha}=\frac{e m}{2 \pi^{2} \hbar^{3}} \int_{0}^{\infty} d E_{\perp} \int_{\Phi}^{\infty} d E_{z}\left[1-r_{l}\left(E_{z}\right)\right] f_{F D}^{\alpha}
$$

and

$$
J_{Q_{\alpha}}=\frac{m}{2 \pi^{2} \hbar^{3}} \int_{0}^{\infty} d E_{\perp} \int_{\Phi}^{\infty} d E_{z} E\left[1-r_{l}\left(E_{z}\right)\right] f_{F D}^{\alpha}
$$

where $f_{F D}^{\alpha}=f_{F D}\left(E, \mu_{\alpha}, T_{\alpha}\right)$ is the Fermi Dirac distribution function for the electrons in electrode $\alpha(=l, r), E=E_{\perp}+E_{z}, E_{z}$ is the energy for the momentum in the $\mathrm{z}$-direction necessary to surmount the barrier, $E_{\perp}$ is the energy for the momentum in the plane perpendicular to z-direction, and $\mu_{\alpha}$ is the chemical potentials as shown in Fig. 1.

The total electric current $J$ and the total thermal current $J_{Q}$ are given by

$$
J=J_{l}-J_{r}
$$

and

$$
J_{Q}=J_{Q_{l}}-J_{Q r}-\frac{T_{r}-T_{l}}{R_{l}} .
$$

The last term in Eq. (9) denotes the heat flow from hot(right) side to cold(left) side, and $R_{l}$ is the lattice thermal resistance.

\section{B. Comparison between models with and without the ROB effect}

From Eq. (1), the COP with the ROB effect is given as

$$
\mathrm{COP}_{1}=\frac{J_{Q}}{v J}
$$

where $Q_{1}=J_{Q}$ is the extracted heat per unit area from the cold electrode, $v$ is the external bias, $W=v J$ is the work done per unit area by the external source, $J$ is the charge current density, $J$ and $J_{Q}$ are given by Eqs. (8) and (9), respectively.

We now focus on the ROB effect. The result without the ROB effect can be obtained by letting $r_{l}=0$ in Eqs. (6) and (7). We denote $J_{M_{\alpha}}=J_{\alpha}\left(r_{l}=0\right), J_{Q_{M_{\alpha}}}=J_{Q_{\alpha}}\left(r_{l}=0\right)$, as the electrical and thermal currents in the absence of ROB effect. The corresponding COP is

$$
\mathrm{COP}_{0}=\frac{J_{Q, M}}{v J_{M}},
$$

where $J_{Q, M}=J_{Q_{M_{l}}}-J_{Q_{M_{r}}}-\left(T_{r}-T_{l}\right) / R_{l}$, and $J_{M}=J_{M_{l}}-J_{M_{r}}$.

Figure 2 shows the bias dependent $\mathrm{COP}_{0}$ and $\mathrm{COP}_{1}$ under certain parameters. Figure 2(a) shows that there exists a critical value of bias when bias is smaller (larger) than the critical value $\mathrm{COP}_{0}>\mathrm{COP}_{1}\left(\mathrm{COP}_{1}>\mathrm{COP}_{0}\right)$. The critical bias is absent in Fig. 2(b). The result indicates that (i) the ROB effect can increase the COP in some cases and (ii) there exist a minimum value of $R_{l}$ for $\mathrm{COP}_{1}>\mathrm{COP}_{0}$ to be possible. The first result is due to the fact that the transmission asymmetry increases with the bias. 

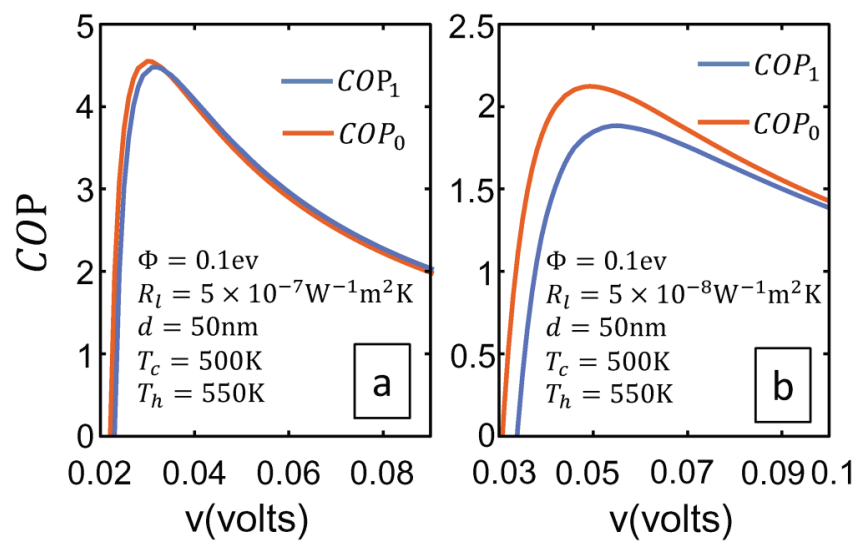

FIG. 2. Plot of COP vs the bias voltage. (a) $R_{l}=5 \times 10^{-7} \mathrm{~W}^{-1} \mathrm{~m}^{2} \mathrm{~K}$ and (b) $R_{l}=5 \times 10^{-8} \mathrm{~W}^{-1} \mathrm{~m}^{2} \mathrm{~K} . \mathrm{COP}_{0}$ and $\mathrm{COP}_{1}$ stand for the result from the model with and without the ROB effect, respectively.

This leads to a larger net thermal and electric current from the cold to the hot electrodes. As a result, the $\mathrm{COP}_{1}>\mathrm{COP}_{0}$ at higher bias. The second result on the $R_{l}$ dependence can be further analyzed. By letting $\mathrm{COP}_{1}>\mathrm{COP}_{0}$, we obtain

$$
R_{l}>R_{\min }^{v}=\frac{J_{M}-J}{J_{Q 1} J_{M}-J_{Q, M 1} J} \delta T
$$

where $\delta T=T_{r}-T_{l}, J_{Q 1}=J_{Q_{l}}-J_{Q_{r}}$, and $J_{Q, M 1}=J_{Q_{M_{l}}}-J_{Q_{M_{r}}}$. The larger the $R_{\min }^{v}$, the more difficult to achieve $\mathrm{COP}_{1}>\mathrm{COP}_{0}$.

Figure 3 plots $R_{\min }^{v}$ against bias voltage $v$. As bias voltage increases, $R_{\min }^{v}$ decreases. The origin of the $R_{l}$ dependence is that the ROB effect makes $J$ and $J_{Q 1}$ smaller than $J_{M}$ and $J_{Q, M 1}$. The COP reduction due to the thermal resistance is different between
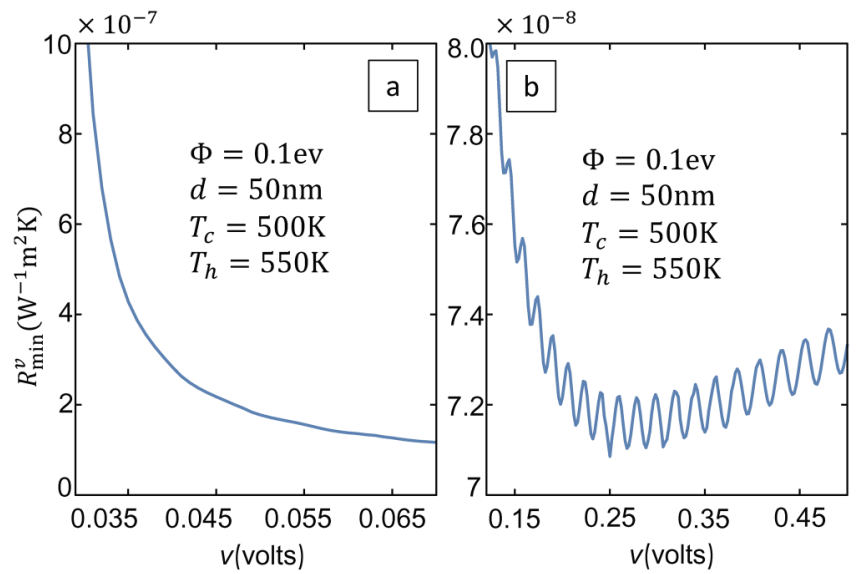

FIG. 3. $R_{\min }^{v}$ plots against the applied bias. When bias is low, $R_{\min }^{v}$ decreases when bias voltage increases as shown in (a), but when bias is high, $R_{\min }^{v}$ increases when bias voltage increases as shown in (b). the model without the ROB effect and the model with the ROB effect. For a certain value of $R_{l}$, say, $5 \times 10^{-7} \mathrm{~W}^{-1} \mathrm{~m}^{2} \mathrm{~K}$ as used in plotting Fig. 2(a), the corresponding critical bias is around $0.033 \mathrm{~V}$, which is slightly greater than the optimum bias of $\mathrm{COP}_{0}$ (around $0.03 \mathrm{~V}$ ). For bias greater than $0.033 \mathrm{~V}, \mathrm{COP}_{1}>\mathrm{COP}_{0}$. If we can further increase $R_{l}$ to make the critical value of bias less than $0.03 \mathrm{~V}$ and keep other parameters unchanged, $\mathrm{COP}_{1}>\mathrm{COP}_{0}$ can be achieved even for optimum $\mathrm{COP}_{0}$.

$R_{\min }^{v}$ has a quite different bias dependence under large bias as shown in Fig. 3(b). This is because under a high bias, the thermionic emission is mainly from the carriers with energy close to the workfunction. The number of carriers with higher energy decreases rapidly and their contribution is negligible. Under a high bias, the structure is more asymmetric and the reflection increases, which results in a smaller electrical and thermal current. Furthermore, the $R_{\min }^{v}$ exhibits a profound quantum oscillation under high bias. Under high bias, the net current is dominated by the current emitted from the left electrode. The reflection coefficient is an oscillatory function of the bias which is manifested in $R_{\min }^{v}$. Under a low bias, this quantum oscillation is suppressed by the current emitted from the opposite electrodes.

\section{Figure of merit}

The $Z T$ of a thermionic device can be analyzed by making an analogy with a thermoelectric device. For small bias voltage and small temperature difference $\delta T=T_{h}-T_{c}$, where $T_{c}=T_{l}$ and $T_{h}=T_{r}, J$ and $J_{Q}$ can be expressed as

$$
J=\mathcal{L}_{11} v+\mathcal{L}_{12}\left(-\frac{\delta T}{T}\right)
$$

and

$$
J_{Q}=\mathcal{L}_{21} v+\left(\mathcal{L}_{22}+\frac{T}{R_{l}}\right)\left(-\frac{\delta T}{T}\right)
$$

where the coefficients $\mathcal{L}_{i j}$ are given as

$$
\mathcal{L}_{i j}=\frac{e^{4-i-j} m}{2 \pi^{2} \hbar^{3}} \int_{0}^{\infty} d E_{\perp} \int_{\Phi}^{\infty} d E_{z} e^{-\frac{E}{k_{B} T}} \frac{E^{(i+j-2)}}{k_{B} T}(1-g) .
$$

Here,

$$
\begin{aligned}
T & =\frac{T_{c}+T_{h}}{2}, \\
k_{\Phi} & =\sqrt{\frac{2 m\left(E_{z}-\Phi\right)}{\hbar^{2}}}, \\
c_{1} & =2\left(\frac{k_{\Phi}^{2}}{k_{1}}-k_{1}\right) \sin \left(k_{\Phi} d\right), \\
c_{2} & =-2\left(\frac{k_{\Phi}^{2}}{k_{1}}+k_{1}\right) \sin \left(k_{\Phi} d\right)-4 i k_{\Phi} \cos \left(k_{\Phi} d\right), \\
g & =\frac{\left|c_{1}\right|^{2}}{\left|c_{2}\right|^{2}} .
\end{aligned}
$$


The corresponding thermoelectric quantities are found as ${ }^{42}$

$$
\begin{gathered}
\sigma=\mathcal{L}_{11} d, \\
S=\frac{\mathcal{L}_{12}}{\mathcal{L}_{11} T}, \\
\kappa=\left[\frac{\mathcal{L}_{22} \mathcal{L}_{11}-\left(\mathcal{L}_{12}\right)^{2}}{\mathcal{L}_{11} T}+\frac{1}{R_{l}}\right] d
\end{gathered}
$$

and

$$
(Z T)_{1}=\frac{\sigma S^{2} T}{\kappa}=\frac{\left(\mathcal{L}_{12}\right)^{2}}{\mathcal{L}_{11} \mathcal{L}_{22}-\left(\mathcal{L}_{12}\right)^{2}+e T \mathcal{L}_{11} / R_{l}} .
$$

We can make a comparison between results with and without the ROB effect. The relevant quantities $\left[\sigma_{M}, S_{M}, \kappa_{M},(Z T)_{0}\right]$ in the absence of ROB have the same form as those in Eqs. (19)-(22) and the coefficients of linear response $\mathcal{L}_{i j}^{M}$ is given as

$$
\mathcal{L}_{i j}^{M}=\frac{e^{4-i-j} m}{2 \pi^{2} \hbar^{3}} \int_{0}^{\infty} d E_{\perp} \int_{\Phi}^{\infty} d E_{z} e^{-\frac{E}{k_{B} T}} \frac{E^{(i+j-2)}}{k_{B} T} .
$$

Because the ROB correction factor $1-g$ is limited in the range of $0 \leq 1-g \leq 1, \mathcal{L}_{i j}^{M}$ is always greater than $\mathcal{L}_{i j}$, i.e., $\mathcal{L}_{i j}^{M}>\mathcal{L}_{i j}$. By varying the bias voltage, we find the maximum COP, given as

$$
C O P_{\max }=\frac{Z T}{(1+\sqrt{1+Z T})^{2}} .
$$

The ROB effect on $\mathrm{COP}_{\max }$ is contained in the ROB-dependent $Z T$.

\section{RESULTS AND DISCUSSION}

We have numerically calculated the $Z T$ for various temperatures and thermal resistance values. The ROB effect is dependent on the barrier width $d$. To minimize both the tunneling and scattering, $d$ is longer than the tunneling length and shorter than the electron mean free path (MFP). The tunneling length is around $5-10 \mathrm{~nm}$ for most semiconductors and the MFP is in the order of $100 \mathrm{~nm}$. We used $d=50 \mathrm{~nm}$ in our calculations.

Figure 4 depicts $(Z T)_{0}$ and $(Z T)_{1}$ for several sets of parameters. We make the following observations. (i) There is a critical workfunction, below this critical workfunction, $(Z T)_{1}>(Z T)_{0}$ as shown in the insets of Fig. 4 . In this situation, the $\mathrm{COP}_{1}$ can be optimized to be greater than $\mathrm{COP}_{0}$. (ii) The optimum $\Phi$ is lower in the presence of ROB. This implies that the ROB effect can reduce the required workfunction where the maximum $Z T$ occurs. (iii) The optimum $(Z T)_{1}$ is always smaller than optimum $(Z T)_{0}$. For example, the optimum $(Z T)_{1}$ is about 6.5 for parameters with $R_{l}=5 \times 10^{-7} \mathrm{~W}^{-1} \mathrm{~m}^{2} \mathrm{~K}$ and $T=500 \mathrm{~K}$ as shown in Fig. 4 (a) with orange color, while the optimum $(Z T)_{0}$ is about 7.1 for same parameters, leading to a reduction of about $8 \%$. If we optimize workfunction and bias at same time, the ROB effect will reduce the overall optimum $\mathrm{COP}$.
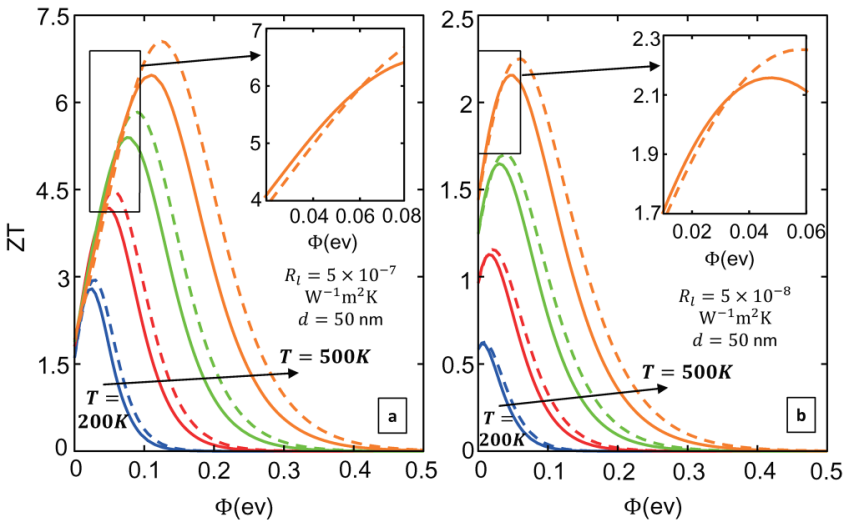

FIG. 4. $(Z T)_{0}$ (dashed line) and $(Z T)_{1}$ (solid line) plot against $\Phi$ at $d=50 \mathrm{~nm}$, temperatures with $200 \mathrm{~K}, 300 \mathrm{~K}, 400 \mathrm{~K}$, and $500 \mathrm{~K}$, according to the arrow direction in the bottom and (a) $R_{l}=5 \times 10^{-7} \mathrm{~W}^{-1} \mathrm{~m}^{2} \mathrm{~K}$, (b) $R_{\mathrm{l}}=5 \times 10^{-8} \mathrm{~W}^{-1} \mathrm{~m}^{2} \mathrm{~K}$. Insets specify when $(Z T)_{0}$ is greater than $(Z T)_{1}$, for simplicity we only show the curve with $T=500 \mathrm{~K}$, this behavior exists at all temperatures plot here just with different regions of $\Phi$. $(Z T)_{0}$ and $(Z T)_{1}$ are the results determined from the model without and with the ROB effect, respectively.

The condition for $(Z T)_{1}>(Z T)_{0}$ is given as

$$
\frac{\left(\mathcal{L}_{12}\right)^{2}}{\mathcal{L}_{11} \mathcal{L}_{22}-\left(\mathcal{L}_{12}\right)^{2}+e T \mathcal{L}_{11} / R_{l}}>\frac{\left(\mathcal{L}_{12}^{M}\right)^{2}}{\mathcal{L}_{11}^{M} \mathcal{L}_{22}^{M}-\left(\mathcal{L}_{12}^{M}\right)^{2}+e T \mathcal{L}_{11}^{M} / R_{l}} .
$$

We denote $R_{\min }^{\Phi}$ as the minimum thermal resistance which satisfies the condition

$$
\begin{aligned}
\frac{e T}{R_{\text {min }}^{\Phi}}\left[\mathcal{L}_{11}^{M}\left(\mathcal{L}_{12}\right)^{2}-\mathcal{L}_{11}\left(\mathcal{L}_{12}^{M}\right)^{2}\right]= & \left(\mathcal{L}_{12}^{M}\right)^{2}\left[\mathcal{L}_{11} \mathcal{L}_{22}-\left(\mathcal{L}_{12}\right)^{2}\right] \\
& -\left(\mathcal{L}_{12}\right)^{2}\left[\mathcal{L}_{11}^{M} \mathcal{L}_{22}^{M}-\left(\mathcal{L}_{12}^{M}\right)^{2}\right] .
\end{aligned}
$$

In Fig. 5(a), we show how $R_{\min }^{\Phi}$ varies with $\Phi$ at $T=300 \mathrm{~K}$. As $\Phi$ increases, $R_{l}$ increases exponentially and rapidly approaches a very large value. If we choose a typical value of lattice thermal conductivity as $1 \mathrm{Wm}^{-1} \mathrm{~K}^{-1}$, then $R_{l}=5 \times 10^{-8} \mathrm{~W}^{-1} \mathrm{~m}^{2} \mathrm{~K}$. The corresponding $\Phi$ is around $0.01 \mathrm{~V}$, which is quite small. If we can increase $R_{l}$, we can relax the restriction of $\Phi$. But if the workfunction is too high, $R_{\min }^{\Phi}$ becomes negative as shown in Fig. 5(b). Therefore, it is impossible for $(Z T)_{1}>(Z T)_{0}$ when workfunction is too high. This is shown in Fig. 4 , where $(Z T)_{0}>(Z T)_{1}$ under a large workfunction $\Phi$.

In conclusion, the ROB effect in a single barrier thermionic cooling device has been investigated. It is found that the COP in the presence of $\mathrm{ROB}$ can be higher than that in the absence of $\mathrm{ROB}, \mathrm{COP}_{1}>\mathrm{COP}_{0}$, if the bias voltage $v$ and the lattice thermal resistance $R_{l}$ satisfy relation Eq. (12). $Z T$ is higher in the presence of the ROB effect, $(Z T)_{1}>(Z T)_{0}$, if the workfunction $\Phi$ and the lattice thermal resistance $R_{l}$ satisfy relation Eq. (20). The optimum $(Z T)_{1}$ is smaller than optimum $(Z T)_{0}$. These results can be useful in designing thermionic devices. 


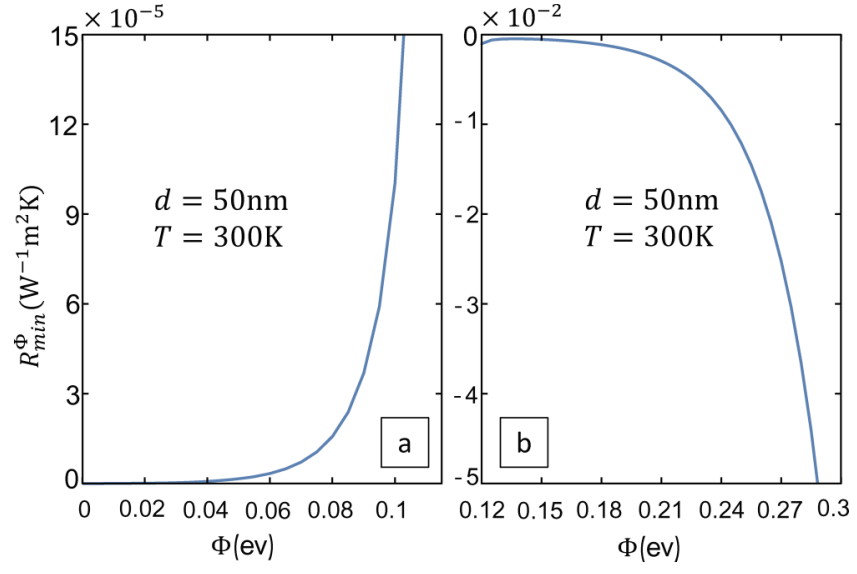

FIG. 5. The minimum $R_{l}$ vs the workfunction $\Phi$. When $\Phi$ is not very large, $R_{\min }^{\phi}$ increases when $\Phi$ increases as shown in (a). In (b), it is shown that as $\Phi$ is increased to a large value, $R_{\min }^{\phi}$ becomes negative.

\section{ACKNOWLEDGMENTS}

We thank Peng Jiang and Yee Sin Ang for useful discussions, and acknowledge support from NSFC (No. 11774006), NBRP of China (No. 2012CB921300), and the Australian Research Council Grant (No. DP160101474).

\section{DATA AVAILABILITY}

The data that support the findings of this study are available within the article.

\section{REFERENCES}

'E. Mykkänen, J. S. Lehtinen, L. Grönberg, A. Shchepetov, A. V. Timofeev, D. Gunnarsson, A. Kemppinen, A. J. Manninen, and M. Prunnila, "Thermionic junction devices utilizing phonon blocking," Sci. Adv. 6, eaax9191 (2020).

${ }^{2}$ Y. S. Ang, Y. Chen, C. Tan, and L. K. Ang, "Generalized high-energy thermionic electron injection at graphene interface,” Phys. Rev. Appl. 12, 014057 (2019).

${ }^{3} \mathrm{M}$. Trushin, "Theory of photoexcited and thermionic emission across a twodimensional graphene-semiconductor Schottky junction," Phys. Rev. B 97, 195447 (2018).

${ }^{4}$ S. Huang, M. Sanderson, Y. Zhang, and C. Zhang, "High efficiency and non-Richardson thermionics in three dimensional Dirac materials," Appl. Phys. Lett. 111, 183902 (2017).

${ }^{5}$ S. Huang, R. A. Lewis, and C. Zhang, "Thermionic enhanced heat transfer in electronic devices based on 3D Dirac materials," J. Appl. Phys. 126, 165105 (2019).

${ }^{6}$ Y. S. Ang and L. K. Ang, "Theory of thermionic carrier injection in graphene/ organic Schottky interface,” Front. Mater. 27, 00204 (2019).

${ }^{7}$ S. Madas, S. K. Mishra, S. Kahaly, and M. U. Kahaly, "Superior photothermionic electron emission from illuminated phosphorene surface," Sci. Rep. 9, 10307 (2019).

${ }^{8}$ M. Massicotte, P. Schmidt, F. Vialla, K. Watanabe, T. Taniguchi, K. J. Tielrooij, and F. H. L. Koppens, "Photo-thermionic effect in vertical graphene heterostructures," Nat. Commun. 7, 12174 (2016).

${ }^{9} \mathrm{M}$. Zebarjadi, "Solid-state thermionic power generators: An analytical analysis in the nonlinear regime," Phys. Rev. Appl. 8, 014008 (2017).
${ }^{10} \mathrm{~S} . \mathrm{Wu}, \mathrm{N}$. Hogan, and M. Sheldon, "Hot electron emission in plasmonic thermionic converters," ACS Energy Lett. 4, 2508 (2019).

${ }^{11}$ I. T. Lim, S. A. Lambert, J.-L. Vay, and J. W. Schwede, "Electron reflection in thermionic energy converters," Appl. Phys. Lett. 112, 073906 (2018).

${ }^{\mathbf{1 2}}$ H. M. Nguyen, J. Lu, H. Gotob, and R. Maeda, "Thermionic emission via a nanofluid for direct electrification from low-grade heat energy," Nano Energy 49, 1732 (2018).

${ }^{13}$ D. C. Riley, Z.-X. Shen, P. A. Pianett, N. A. Melosh, and R. T. Howe, "Back-gated graphene anode for more efficient thermionic energy converters," Nano Energy 32, 67 (2017).

${ }^{14}$ G. D. Mahan, “Thermionic refrigeration," J. Appl. Phys. 76, 4362 (1994).

${ }^{15}$ G. D. Mahan, J. O. Sofo, and M. Bartkowiak, "Multilayer thermionic refrigerator and generator,” J. Appl. Phys. 8, 4683 (1998).

${ }^{16}$ G. D. Mahan and L. M. Woods, "Multilayer thermionic refrigeration," Phys. Rev. Lett. 80, 4016 (1998).

${ }^{17}$ A. Shakouri and J. E. Bowers, "Heterostructure integrated thermionic coolers," Appl. Phys. Lett. 71, 1234 (1997).

${ }^{18}$ J. Zhang, N. G. Anderson, and K. M. Lau, "AlGaAs superlattice microcoolers," Appl. Phys. Lett. 83, 374 (2003).

${ }^{19}$ X. Fan, G. Zeng, C. LaBounty, J. E. Bowers, E. Croke, C. C. Ahn, S. Huxtable, A. Majumdar, and A. Shakouri, "SiGeC/Si superlattice microcoolers," Appl. Phys. Lett. 78, 1580 (2001).

${ }^{20}$ G. Zeng, X. Fan, C. LaBounty, E. Croke, Y. Zhang, J. Christofferson, D. Vashaee, A. Shakouri, and J. E. Bowers, "Cooling power density of SiGe/Si superlattice micro refrigerators," MRS Proc. 793, S2.2 (2004).

${ }^{21}$ A. Shakouri, C. LaBounty, P. Abraham, J. Piprek, and J. E. Bowers, "Enhanced thermionic emission cooling in high barrier superlattice heterostructures," MRS Proc. 545, 449 (1999).

${ }^{22}$ R. Singh, D. Vashaee, Y. Zhang, M. Negassi, A. Shakouri, Y. Okuno, G. Zeng, C. LaBounty, and J. Bowers, "Experimental characterization and modeling of InP-based microcoolers," MRS Proc. 793, S11.4 (2004).

${ }^{23}$ M. Kardar, Statistical Physics of Particles (Cambridge University Press, 2007).

${ }^{24}$ K. Sahasrabuddhe, J. W. Schwede, I. Bargatin, J. Jean, R. T. Howe, Z.-X. Shen, and N. A. Melosh, "A model for emission yield from planar photocathodes based on photonenhanced thermionic emission or negative-electron-affinity photoemission," J. Appl. Phys. 112, 094907 (2012).

${ }^{25}$ J. R. Smith, G. L. Bilbro, and R. J. Nemanich, "Theory of space charge limited regime of thermionic energy converter with negative electron affinity emitter," J. Vac. Sci. Technol. B: Microelectronics Nanometer Struct. 27, 1132 (2009).

${ }^{\mathbf{2 6}} \mathrm{J}$. R. Smith, "Increasing the efficiency of a thermionic engine using a negative electron affinity collector," J. Appl. Phys. 114, 164514 (2013).

${ }^{27}$ X. M. Wang, M. Zebarjadi, and K. Esfarjani, "First principles calculations of solid-state thermionic transport in layered van der Waals heterostructures," Nanoscale 8, 14695 (2016).

${ }^{28}$ S.-J. Liang, B. Liu, W. Hu, K. Zhou, and L. K. Ang, "Thermionic energy conversion based on graphene van der Waals heterostructures," Sci. Rep. 7, 46211 (2017).

${ }^{29}$ X. M. Wang, M. Zebarjadi, and K. Esfarjani, "High performance solid-state thermionic energy conversion based on $2 \mathrm{~d}$ van der Waals heterostructures: A first principles study," Sci. Rep. 8, 9303 (2018).

${ }^{30}$ S. Misra, M. Upadhyay Kahaly, and S. K. Mishra, "Thermionic emission from monolayer graphene, sheath formation and its feasibility towards thermionic converters," J. Appl. Phys. 121, 065102 (2017).

${ }^{31}$ S.-J. Liang and L. K. Ang, "Electron thermionic emission from graphene and a thermionic energy converter," Phys. Rev. Appl. 3, 014002 (2015).

${ }^{32}$ V. Giorgis, F. Morini, T. Zhu, J.-F. Robillard, X. Wallart, J.-L. Codron, and E. Dubois, "Synthesis and characterization of low work function alkali oxide thin films for unconventional thermionic energy converters," J. Appl. Phys. 120, 205108 (2016). 
${ }^{33}$ Y. S. Ang, H. Y. Yang, and L. K. Ang, "Universal scaling laws in Schottky heterostructures based on two-dimensional materials," Phys. Rev. Lett. 121, 056802 (2018).

${ }^{34}$ J. W. Schwede, T. Sarmiento, V. K. Narasimhan, S. J. Rosenthal, D. C. Riley, F. Schmitt, I. Bargatin, K. Sahasrabuddhe, R. T. Howe, J. S. Harris, N. A. Melosh, and Z.-X. Shen, "Photon-enhanced thermionic emission from heterostructures with low interface recombination," Nat. Commun. 4, 1576 (2013).

${ }^{35}$ J. W. Schwede, I. Bargatin, D. C. Riley, B. E. Hardin, S. J. Rosenthal, Y. Sun, F. Schmitt, P. Pianetta, R. T. Howe, Z.-X. Shen, and N. A. Melosh, "Photon-enhanced thermionic emission for solar concentrator systems," Nat. Mater. 9, 762 (2010).

${ }^{36} \mathrm{~K}$. Reck and O. Hansen, "Thermodynamics of photon-enhanced thermionic emission solar cells," Appl. Phys. Lett. 104, 023902 (2014).

${ }^{37}$ M. U. Kahaly, S. Misra, and S. K. Mishra, "Photo-assisted electron emission from illuminated monolayer graphene," J. Appl. Phys. 121, 205110 (2017).
${ }^{38}$ G. Xiao, G. H. Zheng, D. Ni, Q. Li, M. Qiu, and M. J. Ni, "Thermodynamic assessment of solar photon-enhanced thermionic conversion," Appl. Energy 223, 134 (2018).

${ }^{39}$ A. Kribus and G. Segev, "Solar energy conversion with photon-enhanced thermionic emission," J. Opt. 18, 073001 (2016).

${ }^{40} \mathrm{~S}$. Su, X. H. Chen, T. J. Liao, J. C. Chen, and T.-M. Shih, "Photon-enhanced electron tunneling solar cells," Energy 111, 52 (2016).

${ }^{41} \mathrm{H}$. J. Goldsmid, Introduction to Thermoelectricity (Springer, Berlin, Heidelberg, 2016).

${ }^{42}$ N. Mermin and N. W. Ashcroft, Solid State Physics (Cengage Learning Inc, 1976).

${ }^{43}$ S. M. Sze and K. K. Ng, Physics of Semiconductor Devices (John Wiley \& Sons Inc, 2007).

${ }^{44} \mathrm{H}$. J. Weber and G. B. Arfken, Mathematical Methods for Physicists (Elsevier Ltd, Oxford, 2012). 